TRANSACTIONS OF THE

AMERICAN MATHEMATICAL SOCIETY

Volume 361, Number 10, October 2009, Pages 5177-5192

S 0002-9947(09)04621-2

Article electronically published on April 21, 2009

\title{
SHEAVES OF NONLINEAR GENERALIZED FUNCTIONS AND MANIFOLD-VALUED DISTRIBUTIONS
}

\author{
MICHAEL KUNZINGER, ROLAND STEINBAUER, AND JAMES A. VICKERS
}

\begin{abstract}
This paper is part of an ongoing program to develop a theory of generalized differential geometry. We consider the space $\mathcal{G}[X, Y]$ of Colombeau generalized functions defined on a manifold $X$ and taking values in a manifold $Y$. This space is essential in order to study concepts such as flows of generalized vector fields or geodesics of generalized metrics. We introduce an embedding of the space of continuous mappings $\mathcal{C}(X, Y)$ into $\mathcal{G}[X, Y]$ and study the sheaf properties of $\mathcal{G}[X, Y]$. Similar results are obtained for spaces of generalized vector bundle homomorphisms. Based on these constructions we propose the definition of a space $\mathcal{D}^{\prime}[X, Y]$ of distributions on $X$ taking values in $Y . \mathcal{D}^{\prime}[X, Y]$ is realized as a quotient of a certain subspace of $\mathcal{G}[X, Y]$.
\end{abstract}

\section{INTRODUCTION}

Nonlinear generalized functions in the sense of J.F. Colombeau (4, 5, 6]) are an extension of the (linear) theory of distributions providing maximal consistency with respect to classical analysis in light of L. Schwartz's impossibility result ([36]). While originally used as a tool for studying nonlinear partial differential equations (see 35 for a survey), applications of a more geometric nature, in particular, in Lie group analysis of differential equations (e.g. 25, 16, ) and in general relativity (see [37. for a recent review) have led to the development of a geometric theory of nonlinear generalized functions (see [16] for an overview).

According to general relativity the curvature of space-time is given by Einstein's equations, which form a nonlinear system of second order partial differential equations for the metric. In order to have a well-defined space-time one therefore requires sufficient differentiability of the metric for Einstein's equations to make sense. However there are a number of physically important solutions for which the metric does not possess the necessary level of differentiability. Examples of this include spacetimes with pp-waves and cosmic strings. In order to enlarge the class of space-times one can deal with, so that they include those of physical importance, it is necessary to develop a theory of distributional geometry. However because the curvature is a nonlinear function of the derivatives of the components of the metric, such a

Received by the editors April 16, 2007 and, in revised form, August 24, 2007.

2000 Mathematics Subject Classification. Primary 46T30; Secondary 46F30, 53B20.

Key words and phrases. Algebras of generalized functions, Colombeau algebras, generalized functions on manifolds, manifold-valued distributions.

This work was supported by project P16742 and START-project Y-237 of the Austrian Science Fund.

(C)2009 American Mathematical Society 
theory has to be produced using the theory of nonlinear generalized functions. A key step in this program was the construction of a diffeomorphism invariant scalar theory in the so-called 'full' setting where one has a canonical embedding of distributions into the algebra [15, 17, 21, 22]. This built upon the pioneering work of 2, 17, 20. However in order to develop a theory of generalized differential geometry one needs to go beyond this and have a description of generalized tensor fields, a topic of ongoing research. On the other hand for the so-called 'special' setting (which will be the framework of this article) building on [9], a theory of generalized sections in vector bundles was introduced in 27. It extends the distributional theory of De Rham and Marsden ([8, 31] ) and was used to introduce a generalized (pseudo-)Riemannian geometry in 28]. Moreover, the need to consider geodesics of a generalized (pseudo-)Riemannian metric or the flow of a generalized vector field made it essential to consider generalized functions taking values in a manifold (a concept not available in classical distribution theory). To this end, in 24] the space $\mathcal{G}[X, Y]$ of generalized functions defined on a manifold $X$ and taking values in a manifold $Y$ was introduced as well as the space $\operatorname{Hom}_{\mathcal{G}}(E, F)$ of generalized vector bundle homomorphisms. The notions of 24] were used in the description of geodesics in the impulsive gravitational pp-waves of general relativity (see [16, Chapter 5 for an overview) as well as in the study of flows of generalized vector fields (26]). On the other hand in [29] the construction of 24] was completed and turned into a functorial theory. In particular, several global characterizations of the spaces $\mathcal{G}[X, Y]$ and $\operatorname{Hom}_{\mathcal{G}}(E, F)$ were given. In this work we develop this line of research further and consider sheaf properties of these spaces as well as the question of the embedding of 'regular' (e.g. continuous, resp. locally bounded) functions into the respective spaces. Based on this we propose the definition of a space of manifold-valued distributions. Our approach extends the sheaf-theoretic study of real-valued Colombeau generalized functions given in [9, 11, 12, 32]; cf. also [13] for an alternative setting.

In some more detail, in section 2 we prove that $\mathcal{G}\left[{ }_{-}, Y\right]$ and $\operatorname{Hom}_{\mathcal{G}}(,, F)$ are sheaves of sets. Section 3 is devoted to proving that the space $\mathcal{C}(X, Y)$ of continuous functions from $X$ to $Y$ is embedded into $\mathcal{G}[X, Y]$ as well as the analogous statement for vector bundle homomorphisms. Furthermore we discuss the (im)possibility of embedding locally bounded functions into $\mathcal{G}[X, Y]$ and provide an embedding for vector bundle homomorphisms which display a 'more singular' behavior in their fiber component. Finally, in section 4 we propose the introduction of a space $\mathcal{D}^{\prime}[X, Y]$ of 'distributions' taking values in $Y$-a notion which does not exist classically. Inspired by the sequential approach to distribution theory on Euclidean space (see [33, 1, 38]) we utilize our constructions to define a quotient of a certain subspace of $\mathcal{G}[X, Y]$ which serves as an analog to these distributional spaces. We conclude this work by investigating the basic properties of $\mathcal{D}^{\prime}[X, Y]$.

In the remainder of this introduction we recall some notions from [24, 29] which will be needed in the sequel and fix some notation. Our main reference on nonlinear generalized functions is [16.

Throughout this paper $X$ and $Y$ denote smooth Hausdorff manifolds with countable basis of dimensions $m$ and $n$, respectively. We set $I=(0,1]$ and $\mathcal{E}(X):=$ $\mathcal{C}^{\infty}(I \times X, \mathbb{C})$. Elements of $\mathcal{E}(X)$ will as usual be denoted in the form $\left(u_{\varepsilon}\right)_{\varepsilon}$ to emphasize the role of $\varepsilon$ as a (regularization) parameter. Note, however, that here and in what follows we suppose smooth dependence on $\varepsilon$. This additional assumption 
leaves unchanged all properties of the spaces of generalized functions as described in [16, 24, 29], yet it will be crucial for the constructions to follow. Independent reasons for this choice of a base space are certain algebraic simplifications: in order for polynomials with generalized coefficients to only have classical solutions one needs at least continuous dependence on $\varepsilon$; cf. [35], Prop. 12.2. or [3], Prop. 1.10.5. A similar statement holds for solutions of polynomial ordinary differential equations; see [3, Cor. 1.10.9.

The special Colombeau algebra of generalized functions $\mathcal{G}(X)$ on $X$ is defined as the quotient $\mathcal{E}_{M}(X) / \mathcal{N}(X)$ of moderate modulo negligible nets in $\mathcal{E}(X)$. Here the latter notions are defined by (denoting by $\mathcal{P}(X)$ the space of linear differential operators on $X$ )

$$
\begin{aligned}
\mathcal{E}_{M}(X):= & \left\{\left(u_{\varepsilon}\right)_{\varepsilon} \in \mathcal{E}(X): \forall K \Subset X, \forall P \in \mathcal{P}(X) \exists N \in \mathbb{N}:\right. \\
& \left.\sup _{p \in K}\left|P u_{\varepsilon}(p)\right|=O\left(\varepsilon^{-N}\right)\right\}, \\
\mathcal{N}(X):= & \left.\left\{\left(u_{\varepsilon}\right)_{\varepsilon} \in \mathcal{E}_{M}(X): \forall K \Subset X, \forall q \in \mathbb{N}_{0}: \sup _{p \in K}\left|u_{\varepsilon}(p)\right|=O\left(\varepsilon^{q}\right)\right)\right\} .
\end{aligned}
$$

Since we are going to work entirely in the 'special' setting of Colombeau's construction we omit this term henceforth. $\mathcal{G}(-)$ is a fine sheaf of differential algebras with respect to the Lie derivative along smooth vector fields defined by $L_{\xi} u=\left[\left(L_{\xi} u_{\varepsilon}\right)_{\varepsilon}\right]$ ([9, 27]). The construction can be appropriately localized; that is, $u$ is in $\mathcal{G}(X)$ if and only if $u \circ \psi_{\alpha} \in \mathcal{G}\left(\psi_{\alpha}\left(V_{\alpha}\right)\right.$ ) (the local Colombeau algebra on $\psi_{\alpha}\left(V_{\alpha}\right)$ ) for all charts $\left(V_{\alpha}, \psi_{\alpha}\right) . \mathcal{C}^{\infty}(X)$ is a subalgebra of $\mathcal{G}(X)$ and there exist injective sheaf morphisms embedding $\mathcal{D}^{\prime}(X)$, the space of Schwartz distributions on $X$, into $\mathcal{G}(X)$.

The space $\mathcal{G}[X, Y]$ of compactly bounded (c-bounded) generalized Colombeau functions on $X$ taking values in $Y$ is defined similarly as a quotient of the set $\mathcal{E}_{M}[X, Y]$ of moderate, c-bounded maps from $X$ to $Y$ by a certain equivalence relation. However, in the absence of a linear structure in the target space the definition of the respective asymptotics becomes more involved.

\subsection{Definition.}

(a) $\mathcal{E}_{M}[X, Y]$ is defined as the set of all $\left(u_{\varepsilon}\right)_{\varepsilon} \in \mathcal{C}^{\infty}(I \times X, Y)$ satisfying

(i) $\forall K \Subset X \exists \varepsilon_{0}>0 \exists K^{\prime} \Subset Y \forall \varepsilon<\varepsilon_{0}: u_{\varepsilon}(K) \subseteq K^{\prime}$ (c-boundedness).

(ii) $\forall k \in \mathbb{N}$, for each chart $(V, \varphi)$ in $X$, each chart $(W, \psi)$ in $Y$, each $L \Subset V$ and each $L^{\prime} \Subset W$ there exists $N \in \mathbb{N}$ with

$$
\sup _{x \in L \cap u_{\varepsilon}^{-1}\left(L^{\prime}\right)}\left\|D^{(k)}\left(\psi \circ u_{\varepsilon} \circ \varphi^{-1}\right)(\varphi(x))\right\|=O\left(\varepsilon^{-N}\right) .
$$

(b) $\left(u_{\varepsilon}\right)_{\varepsilon}$ and $\left(v_{\varepsilon}\right)_{\varepsilon} \in \mathcal{E}_{M}[X, Y]$ are called equivalent, $\left(u_{\varepsilon}\right)_{\varepsilon} \sim\left(v_{\varepsilon}\right)_{\varepsilon}$, if the following conditions are satisfied:

(i) For all $K \Subset X, \sup _{p \in K} d_{h}\left(u_{\varepsilon}(p), v_{\varepsilon}(p)\right) \rightarrow 0(\varepsilon \rightarrow 0)$ for some (hence every) Riemannian metric $h$ on $Y$.

(ii) $\forall k \in \mathbb{N}_{0} \forall m \in \mathbb{N}$, for each chart $(V, \varphi)$ in $X$, each chart $(W, \psi)$ in $Y$, each $L \Subset V$ and each $L^{\prime} \Subset W$ :

$$
\sup _{\varepsilon}\left\|D^{(k)}\left(\psi \circ u_{\varepsilon} \circ \varphi^{-1}-\psi \circ v_{\varepsilon} \circ \varphi^{-1}\right)(\varphi(x))\right\|=O\left(\varepsilon^{m}\right) .
$$

(c) The space of generalized functions from $X$ to $Y$ is defined as

$$
\mathcal{G}[X, Y]:=\mathcal{E}_{M}[X, Y] / \sim
$$


The following characterization result has been established in [29, Prop. 3.2 and Th. 3.3] and will be repeatedly used throughout this work.

\subsection{Theorem.}

(i) Let $\left(u_{\varepsilon}\right)_{\varepsilon} \in \mathcal{C}^{\infty}(I \times X, Y)$. Then $\left(u_{\varepsilon}\right)_{\varepsilon} \in \mathcal{E}_{M}[X, Y]$ if and only if $\left(f \circ u_{\varepsilon}\right)_{\varepsilon} \in$ $\mathcal{E}_{M}(X)$ for all $f \in \mathcal{C}^{\infty}(Y)$.

(ii) If $\left(u_{\varepsilon}\right)_{\varepsilon},\left(v_{\varepsilon}\right)_{\varepsilon} \in \mathcal{E}_{M}[X, Y]$, then $\left(u_{\varepsilon}\right)_{\varepsilon} \sim\left(v_{\varepsilon}\right)_{\varepsilon}$ if and only if $\left(f \circ u_{\varepsilon}-f \circ v_{\varepsilon}\right)_{\varepsilon} \in$ $\mathcal{N}(X)$ for all $f \in \mathcal{C}^{\infty}(Y)$.

Finally, we turn to the definition of generalized vector bundle homomorphisms (e.g., tangent maps of manifold-valued generalized functions). Vector bundles with base space $X$ will be denoted $\left(E, X, \pi_{X}\right)$. A vector bundle chart $(V, \Phi)$ over a chart $(V, \varphi)$ of $X$ will be written in the form $\Phi(e)=(\varphi(p), \varphi(e)) \in \varphi(V) \times \mathbb{R}^{n^{\prime}}$, where $p=\pi_{X}(e)$. The space of smooth vector bundle homomorphisms from $E$ to $\left(F, Y, \pi_{Y}\right)$ will be called $\operatorname{Hom}(E, F)$. If $f \in \operatorname{Hom}(E, F)$ we write $f: X \rightarrow Y$ for the smooth map induced on bases, i.e., $\pi_{Y} \circ f=f \circ \pi_{X}$. Local vector bundle

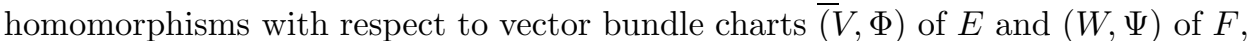
i.e., $f_{\Psi \Phi}:=\Psi \circ f \circ \Phi^{-1}: \varphi\left(V \cap \underline{f}^{-1}(W)\right) \times \mathbb{R}^{m^{\prime}} \rightarrow \psi(W) \times \mathbb{R}^{n^{\prime}}$, will be written in the form

$$
f_{\Psi \Phi}(x, \xi)=\left(f_{\Psi \Phi}^{(1)}(x), f_{\Psi \Phi}^{(2)}(x) \cdot \xi\right) .
$$

Here, $f_{\Psi \Phi}^{(1)}=\underline{f}_{\psi \varphi}:=\psi \circ \underline{f} \circ \varphi^{-1}$. Finally we are ready to define the space of generalized vector bundle homomorphisms.

\subsection{Definition.}

(a) $\mathcal{E}_{M}{ }^{\mathrm{VB}}[E, F]$ is the set of all $\left(u_{\varepsilon}\right)_{\varepsilon} \in \operatorname{Hom}(E, F)^{I}$ depending smoothly on $\varepsilon$ and satisfying

(i) $\left(\underline{u_{\varepsilon}}\right)_{\varepsilon} \in \mathcal{E}_{M}[X, Y]$.

(ii) $\forall k \in \mathbb{N}_{0} \forall(V, \Phi)$ vector bundle chart in $E, \forall(W, \Psi)$ vector bundle chart in $F, \forall L \Subset V \forall L^{\prime} \Subset W \exists N \in \mathbb{N} \exists \varepsilon_{1}>0 \exists C>0$ with

$$
\left\|D^{(k)}\left(u_{\varepsilon \Psi \Phi}^{(2)}(\varphi(p))\right)\right\| \leq C \varepsilon^{-N}
$$

for all $\varepsilon<\varepsilon_{1}$ and all $p \in L \cap \underline{u}_{\varepsilon}^{-1}\left(L^{\prime}\right)$, where $\|\cdot\|$ denotes any matrix norm.

(b) $\left(u_{\varepsilon}\right)_{\varepsilon},\left(v_{\varepsilon}\right)_{\varepsilon} \in \mathcal{E}_{M}{ }^{\mathrm{VB}}[E, F]$ are called vb-equivalent, $\left(\left(u_{\varepsilon}\right)_{\varepsilon} \sim_{v b}\left(v_{\varepsilon}\right)_{\varepsilon}\right)$ if

(i) $\left(\underline{u_{\varepsilon}}\right)_{\varepsilon} \sim\left(\underline{v_{\varepsilon}}\right)_{\varepsilon}$ in $\mathcal{E}_{M}[X, Y]$.

(ii) $\forall k \in \mathbb{N}_{0} \forall m \in \mathbb{N} \forall(V, \Phi)$ vector bundle chart in $E, \forall(W, \Psi)$ vector bundle chart in $F, \forall L \Subset V \forall L^{\prime} \Subset W \exists \varepsilon_{1}>0 \exists C>0$ such that:

$$
\left\|D^{(k)}\left(u_{\varepsilon \Psi \Phi}^{(2)}-v_{\varepsilon \Psi \Phi}^{(2)}\right)(\varphi(p))\right\| \leq C \varepsilon^{m}
$$

for all $\varepsilon<\varepsilon_{1}$ and all $p \in L \cap \underline{u}_{\varepsilon}^{-1}\left(L^{\prime}\right) \cap \underline{v}_{\varepsilon}{ }^{-1}\left(L^{\prime}\right)$.

(c) The space of generalized vector bundle homomorphisms is defined by

$$
\operatorname{Hom}_{\mathcal{G}}[E, F]:=\mathcal{E}_{M}{ }^{\mathrm{VB}}[E, F] / \sim_{v b} .
$$

For $u \in \operatorname{Hom}_{\mathcal{G}}[E, F], \underline{u}:=\left[\left(\underline{u}_{\varepsilon}\right)_{\varepsilon}\right]$ is a well-defined element of $\mathcal{G}[X, Y]$ uniquely characterized by $\underline{u} \circ \pi_{X}=\pi_{Y} \circ u$. The tangent map $T u:=\left[\left(T u_{\varepsilon}\right)_{\varepsilon}\right]$ of any $u \in$ $\mathcal{G}[X, Y]$ is a well-defined element of $\operatorname{Hom}_{\mathcal{G}}[T X, T Y]$. 


\section{The Sheaf property of $\mathcal{G}[X, Y]$ and $\operatorname{Hom}_{\mathcal{G}}(E, F)$}

Our aim in this section is to establish that $\mathcal{G}\left[{ }_{-}, Y\right]$ is a sheaf of sets. Compared to the case of the Colombeau algebra $\mathcal{G}(X)$ (where the sheaf property can basically be derived by lifting the local result $([5], \S 1.3)$ to the manifold $([9,[27]))$ the main obstacle in the present setting is the lack of algebraic structure on the target space $Y$. Given an open cover $\left\{U_{\alpha} \mid \alpha \in A\right\}$ of $X$ it follows directly from the definition (or also from Theorem 1.2 (ii)) that if $u, v \in \mathcal{G}[X, Y]$ and $\left.u\right|_{U_{\alpha}}=\left.v\right|_{U_{\alpha}}$ for all $\alpha \in A$, then $u=v$. The second defining property,

Given a family $\left\{u_{\alpha} \in \mathcal{G}\left[U_{\alpha}, Y\right] \mid \alpha \in A\right\}$ such that $\left.u_{\alpha}\right|_{U_{\alpha} \cap U_{\beta}}=$ $\left.u_{\beta}\right|_{U_{\alpha} \cap U_{\beta}}$ for all $\alpha, \beta$ with $U_{\alpha} \cap U_{\beta} \neq \emptyset$, then there exists some $u \in \mathcal{G}[X, Y]$ with $\left.u\right|_{U_{\alpha}}=u_{\alpha}$ for all $\alpha \in A$,

however, cannot be established similar to the algebra-setting: the tools for gluing together locally defined elements of our quotient spaces (e.g., partitions of unity) are absent in the manifold-valued case. Our strategy therefore will be to first embed the target manifold in some Euclidean space by a Whitney embedding, do the gluing via partitions of unity in the surrounding vector space and then project back onto the target manifold $Y$ using the retraction map of a tubular neighborhood of $Y$. To this end we first provide an alternative characterization of $\mathcal{G}[X, Y]$ in case $Y$ is a submanifold of some $\mathbb{R}^{s}$.

2.1. Definition. Let $Y$ be a submanifold of $\mathbb{R}^{s}$. We define $\tilde{\mathcal{G}}[X, Y]$ to be the subset of $\mathcal{G}(X)^{s}$ consisting of those $u \in \mathcal{G}(X)^{s}$ which possess a representative $\left(u_{\varepsilon}\right)_{\varepsilon}$ satisfying:

(i) $u_{\varepsilon}(X) \subseteq Y$ for all $\varepsilon \in I$.

(ii) For each $K \Subset X$ there exist $\varepsilon_{0}>0$ and $K^{\prime} \Subset Y$ such that $u_{\varepsilon}(K) \subseteq K^{\prime}$ for all $\varepsilon<\varepsilon_{0}$.

2.2. Proposition. Let $X, Y$ be smooth manifolds and let $i: Y \hookrightarrow \mathbb{R}^{s}$ be an embedding of $Y$. Then the push-forward $i_{*}: \mathcal{G}[X, Y] \rightarrow \tilde{\mathcal{G}}[X, i(Y)], i_{*}(u)=i \circ u$ is a bijection which commutes with restrictions to open sets. In particular, if $Y$ is a submanifold of some $\mathbb{R}^{s}$ we may identify $\mathcal{G}[X, Y]$ with $\tilde{\mathcal{G}}[X, Y]$.

Proof. We first note that $i_{*}$ is well-defined: for $\left(u_{\varepsilon}\right)_{\varepsilon} \in \mathcal{E}_{M}[X, Y]$, Theorem 1.2 (i) implies that $\left(i \circ u_{\varepsilon}\right)_{\varepsilon} \in \mathcal{E}_{M}(X)^{s}$. Also, properties (i) and (ii) of Definition 2.1 follow immediately. Suppose now that $\left(u_{\varepsilon}\right)_{\varepsilon} \sim\left(v_{\varepsilon}\right)_{\varepsilon}$ for $\left(u_{\varepsilon}\right)_{\varepsilon},\left(v_{\varepsilon}\right)_{\varepsilon} \in \mathcal{E}_{M}[X, Y]$. Then by Theorem 1.2 (ii) we have that $\left(i_{j} \circ u_{\varepsilon}-i_{j} \circ v_{\varepsilon}\right)_{\varepsilon} \in \mathcal{N}(X)$ for $1 \leq j \leq s$, so $\left[\left(i \circ u_{\varepsilon}\right)_{\varepsilon}\right]=\left[\left(i \circ v_{\varepsilon}\right)_{\varepsilon}\right]$ in $\mathcal{G}(X)^{s}$. Moreover, it follows directly from the definitions that $i_{*}$ commutes with restrictions to open sets.

$i_{*}$ is injective: Suppose that $i_{*}\left(\left[\left(u_{\varepsilon}\right)_{\varepsilon}\right]\right)=i_{*}\left(\left[\left(v_{\varepsilon}\right)_{\varepsilon}\right]\right)$, i.e., $\left(i \circ u_{\varepsilon}\right)_{\varepsilon}-\left(i \circ v_{\varepsilon}\right)_{\varepsilon} \in$ $\mathcal{N}(X)^{s}$. An application of the mean value theorem shows that this entails $\left(g \circ i \circ u_{\varepsilon}\right)_{\varepsilon}-\left(g \circ i \circ v_{\varepsilon}\right)_{\varepsilon} \in \mathcal{N}(X)$ for all $g \in \mathcal{C}^{\infty}(i(Y))$. Since $i: Y \rightarrow i(Y)$ is a diffeomorphism, it follows that any $g \in \mathcal{C}^{\infty}(i(Y))$ is of the form $f \circ i^{-1}$ for some $f \in \mathcal{C}^{\infty}(Y)$. Hence Theorem 1.2 (ii) gives $\left(u_{\varepsilon}\right)_{\varepsilon} \sim\left(v_{\varepsilon}\right)_{\varepsilon}$.

$i_{*}$ is surjective: Let $\tilde{u} \in \tilde{\mathcal{G}}[X, i(Y)]$ with representative $\left(\tilde{u}_{\varepsilon}\right)_{\varepsilon}$ satisfying (i) and (ii) of Definition 2.1. Then $u_{\varepsilon}:=i^{-1} \circ \tilde{u}_{\varepsilon}$ defines (by Theorem 1.2 (i)) an element of $\mathcal{E}_{M}[X, Y]$ whose image under $i_{*}$ is $\tilde{u}$.

After these preparations we can now prove:

2.3. Theorem. $\mathcal{G}[-, Y]$ is a sheaf of sets. 
Proof. By Whitney's embedding theorem (cf. [18 or [30]) there exists an embedding of $Y$ into some $\mathbb{R}^{s}$. Due to Proposition 2.2 and our preparatory statements at the beginning of this section it therefore suffices to suppose that $Y$ is in fact a submanifold of some $\mathbb{R}^{s}$ and to establish property $(*)$ for $\tilde{\mathcal{G}}[X, Y]$. Thus let $\left\{U_{\alpha} \mid \alpha \in A\right\}$ be an open cover of $X$ and let $u^{\alpha} \in \tilde{\mathcal{G}}\left[U_{\alpha}, Y\right]$ such that $\left.u^{\alpha}\right|_{U_{\alpha} \cap U_{\beta}}=\left.u^{\beta}\right|_{U_{\alpha} \cap U_{\beta}}$ for all $\alpha, \beta$ with $U_{\alpha} \cap U_{\beta} \neq \emptyset$. Since $X$ is Hausdorff and second countable, it is $\sigma$-compact, and, in particular, paracompact and Lindelöf. Without loss of generality we may therefore suppose that $A$ is countable and that $\left\{U_{\alpha} \mid \alpha \in A\right\}$ is locally finite. Let $\left\{K_{l} \mid l \in \mathbb{N}\right\}$ be an exhaustive sequence of compact sets in $X$ with $K_{l} \subseteq K_{l+1}^{\circ}$ for all $l$. Again without loss of generality we may suppose that $\left\{U_{\alpha} \mid \alpha \in A\right\}$ is a refinement of $\left\{K_{l}^{\circ} \mid l \in \mathbb{N}\right\}$.

Let $T$ be an open tubular neighborhood of $Y$ in $\mathbb{R}^{s}$ (again see [18, or [30]) and denote by $r: T \rightarrow Y,\left.r\right|_{Y}=i d_{Y}$ the corresponding retraction. Choose a closed tubular neighborhood $T^{\prime} \subseteq T$ and a smooth map $\tilde{r}: \mathbb{R}^{s} \rightarrow \mathbb{R}^{s}$ such that $\left.\tilde{r}\right|_{T^{\prime}}=r$. Let $\left\{\chi_{\alpha} \mid \alpha \in A\right\}$ be a partition of unity with supp $\chi_{\alpha} \Subset U_{\alpha}$ for each $\alpha \in A$. For $\varepsilon \in I$ we set

$$
w_{\varepsilon}:=\tilde{r} \circ\left(\sum_{\alpha \in A} \chi_{\alpha} u_{\varepsilon}^{\alpha}\right) .
$$

Then $\left(w_{\varepsilon}\right)_{\varepsilon} \in \mathcal{E}_{M}\left[X, \mathbb{R}^{s}\right]$. Fix $l \in \mathbb{N}$ and let $\alpha_{1}, \ldots, \alpha_{k}$ be the finitely many indices with $\operatorname{supp} \chi_{\alpha_{i}} \cap K_{l} \neq \emptyset(1 \leq i \leq k)$. Choose $\varepsilon_{l}^{\prime}>0$ and $K_{l}^{\prime} \Subset Y$ such that $u_{\varepsilon}^{\alpha_{i}}\left(\operatorname{supp} \chi_{\alpha_{i}} \cap K_{l}\right) \subseteq K_{l}^{\prime}$ for all $1 \leq i \leq k$ and all $\varepsilon<\varepsilon_{l}^{\prime}$. For each $y \in Y$ choose some $R_{y}>0$ such that the ball $B_{R_{y}}(y)$ of radius $R_{y}$ around $y$ in $\mathbb{R}^{s}$ is contained in $T^{\prime}$. Since $K_{l}^{\prime}$ is compact there exists some $\delta>0$ (the Lebesgue number of the covering $\left\{B_{R_{y}}(y) \cap Y \mid y \in K_{l}^{\prime}\right\}$ of $\left.K_{l}^{\prime}\right)$ such that any subset of $K_{l}^{\prime}$ with diameter less than $\delta$ lies entirely within one $B_{R_{y}}(y)$.

Since $u^{\alpha_{i}}-u^{\alpha_{j}}$ is negligible on $U_{\alpha_{i}} \cap U_{\alpha_{j}}$ (in case this set is nonempty) we may choose some $\varepsilon_{l}<\varepsilon_{l}^{\prime}$ such that $\left|u_{\varepsilon}^{\alpha_{i}}(x)-u_{\varepsilon}^{\alpha_{j}}(x)\right|<\delta$ whenever $x \in K_{l} \cap \operatorname{supp} \chi_{\alpha_{i}} \cap$ $\operatorname{supp} \chi_{\alpha_{j}}$ and $\varepsilon<\varepsilon_{l}(1 \leq i \leq k)$. Hence (using the convexity of $B_{R_{y}}(y)$ ) for each $x \in K_{l}$ and each $\varepsilon<\varepsilon_{l}$ there exists some $y \in K_{l}^{\prime}$ such that

$$
\sum_{\alpha \in A} \chi_{\alpha}(x) u_{\varepsilon}^{\alpha}(x) \in B_{R_{y}}(y) \subseteq T^{\prime} .
$$

Therefore,

$$
w_{\varepsilon}(x)=r \circ\left(\sum_{\alpha \in A} \chi_{\alpha}(x) u_{\varepsilon}^{\alpha}(x)\right) \quad \forall x \in K_{l} \forall \varepsilon<\varepsilon_{l} .
$$

If $\beta \in A$ is such that $U_{\beta} \subseteq K_{l}^{\circ}$ and $\varepsilon<\varepsilon_{l}$, then for each $L \Subset U_{\beta}$ and each $x \in L$ we have

$$
\begin{aligned}
\left|w_{\varepsilon}(x)-u_{\varepsilon}^{\beta}(x)\right| & =\left|r \circ\left(\sum_{\alpha \in A} \chi_{\alpha}(x) u_{\varepsilon}^{\alpha}(x)\right)-r\left(u_{\varepsilon}^{\beta}(x)\right)\right| \\
& \leq\|D \tilde{r}\|_{L^{\infty}\left(\operatorname{ch}\left(K_{l}^{\prime}\right)\right)}\left|\sum_{\alpha \in A} \chi_{\alpha}(x) u_{\varepsilon}^{\alpha}(x)-u_{\varepsilon}^{\beta}(x)\right|
\end{aligned}
$$

where $\operatorname{ch}\left(K_{l}^{\prime}\right)$, the convex hull of $K_{l}^{\prime}$, is itself compact. Since on $L$ the last factor in this estimate vanishes faster than any power of $\varepsilon$ we have $\left.w\right|_{U_{\beta}}=u^{\beta}$ for all $\beta$ with $U_{\beta} \subseteq K_{l}^{\circ}$. 
Choose a smooth function $\eta: X \rightarrow \mathbb{R}$ such that $0<\eta(x) \leq \varepsilon_{l}$ for all $x \in K_{l} \backslash K_{l}^{\circ}$ $\left(K_{0}:=\emptyset\right)$ (see, e.g., [16], Lemma 2.7.3). Moreover, let $\nu: \mathbb{R}^{+} \rightarrow[0,1]$ be a smooth function satisfying $\nu(x) \leq x$ for all $x$ and

$$
\nu(x)= \begin{cases}x & 0 \leq x \leq \frac{1}{2} \\ 1 & x \geq \frac{3}{2}\end{cases}
$$

For $(\varepsilon, x) \in I \times X$ we set $\mu(\varepsilon, x):=\eta(x) \nu\left(\frac{\varepsilon}{\eta(x)}\right)$. Finally, we set $u_{\varepsilon}(x):=w_{\mu(\varepsilon, x)}(x)$ for $(\varepsilon, x) \in I \times X$. Then $(\varepsilon, x) \mapsto u_{\varepsilon}(x) \in \mathcal{C}^{\infty}(I \times X, Y)$ (it is here that we need smooth dependence of representatives on $\varepsilon)$. Furthermore,

$$
\left.u_{\varepsilon}\right|_{K_{l}^{\circ}}=\left.w_{\varepsilon}\right|_{K_{l}^{\circ}} \quad \text { for } \varepsilon \leq \frac{1}{2} \min _{x \in K_{l}} \eta(x), l \in \mathbb{N} .
$$

Therefore, $u=\left[\left(u_{\varepsilon}\right)_{\varepsilon}\right] \in \tilde{\mathcal{G}}[X, Y]$ and $\left.u\right|_{U_{\beta}}=u^{\beta}$ for all $\beta \in A$.

2.4. Remark. The method of gluing via the function $\mu$ in the above proof also allows us to establish the equality of the space of generalized functions taking values in an open subset of $\mathbb{R}^{n}$ as introduced in [2] (with smooth dependence on $\varepsilon$ ) with our setting. In fact, let $\Omega \subseteq \mathbb{R}^{m}$ and $\Omega^{\prime} \subseteq \mathbb{R}^{n}$ be open sets and let $u \in \mathcal{G}(\Omega)^{n}$ such that $u$ possesses a representative $\left(u_{\varepsilon}\right)_{\varepsilon}$ satisfying: $\forall K \Subset \Omega \exists K^{\prime} \Subset \Omega^{\prime} \exists \varepsilon_{0}>0$ such that $u_{\varepsilon}(K) \subseteq K^{\prime}$ for all $\varepsilon<\varepsilon_{0}$. Choose an exhaustive sequence $\left\{K_{l} \mid l \in \mathbb{N}\right\}$ of $\Omega$ and corresponding $K_{l}^{\prime} \Subset \Omega^{\prime}$ and $\varepsilon_{l}$ as above. Then defining $\mu$ as in the proof of Theorem 2.3. $\tilde{u}_{\varepsilon}(x):=u_{\mu(\varepsilon, x)}(x)$ defines a representative of $u$ such that $u_{\varepsilon}(\Omega) \subseteq \Omega^{\prime}$ for all $\varepsilon$. This shows that $\mathcal{G}_{*}\left(\Omega, \Omega^{\prime}\right)$ in the sense of $\left[2\right.$ can be identified with $\mathcal{G}\left[\Omega, \Omega^{\prime}\right]$.

In what follows, we want to utilize Theorem 2.3 to establish the sheaf property of the space $\operatorname{Hom}_{\mathcal{G}}(E, F)$ of generalized vector bundle homomorphisms. To this end we need some preparatory constructions for smooth vector bundle homomorphisms.

Let $f: X \rightarrow Y$ be any smooth map. Then $f$ can naturally be extended to a vector bundle homomorphism $\bar{f} \in \operatorname{Hom}(E, F)$ by defining its action on the fibers of $E$ to be 0 (i.e., any local representative $\bar{f}_{\Psi \Phi}$ of $\bar{f}$ is of the form $\left.(x, v) \mapsto\left(f_{\psi \varphi}(x), 0\right)\right)$. Suppose now that $U, V$ are open subsets of $X$ with $V \subset \bar{V} \Subset U$ and let $u \in$ $\operatorname{Hom}\left(\left.E\right|_{U}, F\right)$ be such that $\underline{u}=\left.f\right|_{U}$. Choose a bump function $\chi \in \mathcal{C}^{\infty}(X)$ such that $\operatorname{supp} \chi \Subset U$ and $\left.\chi\right|_{V} \equiv 1$. Then $\chi \cdot u:=e \mapsto \chi\left(\pi_{X}(e)\right) u(e)$ (fiber-wise product) defines an element of $\operatorname{Hom}\left(\left.E\right|_{U}, F\right)$. Moreover, there is a unique element $v=\chi \bullet_{f} u$ of $\operatorname{Hom}(E, F)$ such that $\left.v\right|_{U}=\chi \cdot u$ and $\left.v\right|_{X \backslash U}=\left.\bar{f}\right|_{X \backslash U}$. Then $\left.v\right|_{V}=\left.u\right|_{V}$. We will use this notation in the proof of the following result.

2.5. Theorem. $\operatorname{Hom}_{\mathcal{G}}\left(\pi_{X}^{-1}\left({ }_{-}\right), F\right)$ is a sheaf of sets on $X$.

Proof. As in the case of manifold-valued generalized functions, it follows directly from the definitions that if $\left\{U_{\alpha} \mid \alpha \in A\right\}$ is an open cover and $u, v \in \operatorname{Hom}_{\mathcal{G}}(E, F)$ are such that $\left.u\right|_{U_{\alpha}}=\left.v\right|_{U_{\alpha}}$ for all $\alpha \in A$, then $u=v$. (Here and in what follows we abbreviate $\left.u\right|_{\pi_{X}^{-1}\left(U_{\alpha}\right)}$ by $\left.u\right|_{U_{\alpha}}$.)

Suppose that $\left\{u_{\alpha} \in \operatorname{Hom}\left(\left.E\right|_{U_{\alpha}}\right) \mid \alpha \in A\right\}$ forms a coherent family; i.e., $\left.u_{\alpha}\right|_{U_{\alpha} \cap U_{\beta}}$ $=\left.u_{\beta}\right|_{U_{\alpha} \cap U_{\beta}}$ for all $\alpha, \beta$ with $U_{\alpha} \cap U_{\beta} \neq \emptyset$. Then $\left\{\underline{u}_{\alpha} \mid \alpha \in A\right\}$ forms a coherent family in $\mathcal{G}[X, Y]$, so by Theorem 2.3 there exists a unique element $w \in \mathcal{G}[X, Y]$ such that $\left.w\right|_{U_{\alpha}}=\underline{u}_{\alpha}$ for all $\alpha \in A$. Thus each $u_{\alpha}$ is an element of $\operatorname{Hom}_{w}\left(\left.E\right|_{U_{\alpha}}, F\right)$, the space of generalized vector bundle homomorphisms with base component $w$ (cf. 29], Sec. 5). Then by 29], Prop. 5.7, for each $\alpha \in A$ we may choose a representative $\left(u_{\varepsilon}^{\alpha}\right)_{\varepsilon}$ of $u_{\alpha}$ such that $\underline{u}_{\varepsilon}^{\alpha}=\left.w_{\varepsilon}\right|_{U_{\alpha}}$ for all $\varepsilon \in I$. (We note that in order to adapt the 
proof of Prop. 5.7 in 29] to the present setting of smooth $\varepsilon$-dependence, a 'gluing function' $\mu$ as in the proof of Theorem 2.3 has to be employed.)

Choose now a partition of unity $\left\{\chi_{j} \mid j \in \mathbb{N}\right\}$ subordinate to $\left\{U_{\alpha} \mid \alpha \in A\right\}$ such that $\operatorname{supp} \chi_{j} \Subset U_{\alpha_{j}}$ for each $j$. For each $\varepsilon \in I$ we define $u_{\varepsilon}$ as the following (locally finite) fiber-wise sum:

$$
u_{\varepsilon}:=\sum_{j \in \mathbb{N}} \chi_{j} \bullet_{w_{\varepsilon}} u_{\varepsilon}^{\alpha_{j}} .
$$

Then $u=\left[\left(u_{\varepsilon}\right)_{\varepsilon}\right] \in \operatorname{Hom}_{\mathcal{G}}(E, F)$ and $\underline{u}=w$. By [29], Th. 4.2, in order to show that $\left.u\right|_{U_{\alpha}}=u_{\alpha}$ it suffices to establish Definition 1.3 (b) (ii) for $k=0$ (i.e., we do not have to take into account derivatives). This, however, is immediate from the coherence of the family $\left\{u_{\alpha} \mid \alpha \in A\right\}$ and the fact that $\left\{\chi_{j} \mid j \in \mathbb{N}\right\}$ is a partition of unity.

\section{EMBEDDings}

Our aim in this section is to construct embeddings of spaces of continuous (resp. even more singular) mappings into spaces of manifold-valued generalized functions. The basic idea (similar to a procedure introduced in [34, Part A) is to employ a Whitney embedding of the target space into some $\mathbb{R}^{s}$ and then use convolution for smoothing. The retraction map of a tubular neighborhood of $Y$ in $\mathbb{R}^{s}$ will then be used to project the resulting nets of smooth functions back to $Y$.

As was already pointed out in 24, there is a canonical embedding $\sigma$ of $\mathcal{C}^{\infty}(X, Y)$ into $\mathcal{G}[X, Y], \sigma: u \rightarrow\left[(u)_{\varepsilon}\right]$. The following result extends this embedding to the space of continuous mappings from $X$ to $Y$.

3.1. Theorem. There exists an embedding $\iota: \mathcal{C}(X, Y) \hookrightarrow \mathcal{G}[X, Y]$ with the following properties:

(i) $\iota$ is a sheaf morphism.

(ii) $\left.\iota\right|_{\mathcal{C}^{\infty}(X, Y)}=\sigma$.

(iii) $\iota(u)_{\varepsilon}$ converges to $u$ uniformly on compact sets.

Proof. Using a Whitney embedding of $Y$ in some $\mathbb{R}^{s}$, Proposition 2.2 and the above remarks show that without loss of generality we may suppose that $Y$ is a submanifold of $\mathbb{R}^{s}$ and that it suffices to embed $\mathcal{C}(X, Y)$ into $\tilde{\mathcal{G}}[X, Y]$.

By [27, Th. 1 (or [16], Th. 3.2.10, see also [9, $\S 15$ for an equivalent construction based on de Rham regularizations) there exists an injective sheaf morphism $\tilde{\iota}$ : $\mathcal{D}^{\prime}\left(X, \mathbb{R}^{s}\right) \hookrightarrow \mathcal{G}(M)^{s}$. This embedding is based on regularization via convolution with a mollifier in charts of a given atlas, patched together through a partition of unity. In this way, the convergence properties of the respective regularizations of continuous mappings are preserved. In particular, $\tilde{\iota}(u)_{\varepsilon}$ converges uniformly on compact sets to $u$ for $u \in \mathcal{C}\left(X, \mathbb{R}^{s}\right)$.

In what follows we use the notation of the proof of Theorem 2.3. Let $u \in \mathcal{C}(X, Y)$. For each $l \in \mathbb{N}$ we choose $\varepsilon_{l}>0$ such that $\tilde{\iota}(u)_{\varepsilon}\left(K_{l}\right) \subseteq T^{\prime}$ for all $\varepsilon<\varepsilon_{l}$. Then with $\mu$ chosen with respect to this sequence $\varepsilon_{l}$ we define

$$
\iota(u)_{\varepsilon}(x):=r \circ \tilde{\iota}(u)_{\mu(\varepsilon, x)}(x) .
$$

Then clearly $\iota(u) \in \tilde{\mathcal{G}}[X, Y]$ and $\iota$ commutes with restrictions. Injectivity of $\iota$ follows from the fact that $\iota(u)_{\varepsilon} \rightarrow u$ uniformly on compact sets for $u \in \mathcal{C}(X, Y)$. Indeed, 
for each $l \in \mathbb{N}$ we may choose some $K_{l}^{\prime} \Subset Y$ such that $\iota(u)_{\varepsilon}\left(K_{l}\right) \cup u\left(K_{l}\right) \subseteq K_{l}^{\prime}$ for $\varepsilon$ sufficiently small. For such an $\varepsilon$ and all $x \in K_{l}$ we therefore have

$$
\left|\iota(u)_{\varepsilon}(x)-u(x)\right|=\left|r\left(\tilde{\iota}(u)_{\varepsilon}(x)\right)-r(u(x))\right| \leq\|D \tilde{r}\|_{L^{\infty}\left(\operatorname{ch}\left(K_{l}^{\prime}\right)\right)}\left|\tilde{\iota}(u)_{\varepsilon}(x)-u(x)\right|,
$$

which gives the result. An analogous calculation, based on the fact that $\left.\tilde{l}\right|_{\mathcal{C}^{\infty}\left(X, \mathbb{R}^{s}\right)}$ $=\sigma$ establishes that $\iota_{\mathcal{C}^{\infty}(X, Y)}=\sigma$.

3.2. Remark. At first sight it would seem that an alternative, more direct proof of Theorem 3.1 could be carried out using Theorem 2.3. for open subsets $U, V$ of $\mathbb{R}^{m}$, resp. $\mathbb{R}^{n}$, an embedding of $\mathcal{C}(U, V)$ into $\mathcal{G}[U, V]$ can be achieved by modifying the embedding $\iota^{n}$ of $\mathcal{D}^{\prime}(U)^{n}$ into $\mathcal{G}(U)^{n}$ (cf., e.g., [16], Th. 1.2.20) using a compact exhaustion of $U$ and a gluing procedure as in Remark 2.4. together with the fact that $\iota^{n}(u)_{\varepsilon}$ converges to $u$ uniformly on compact sets. In this way, for each $u \in \mathcal{C}(X, Y)$ one can construct a family of embeddings of $\left.u\right|_{U_{\alpha}}$ into $\mathcal{G}\left[U_{\alpha}, Y\right]$ for a covering $\left\{U_{\alpha} \mid \alpha \in A\right\}$ of $X$ by chart domains. Note, however, that the resulting family in general is not coherent: in fact, this would require the standard embedding $\iota: \mathcal{C} \rightarrow \mathcal{G}$ to satisfy $f \circ \iota(u) \circ g=\iota(f \circ u \circ g)$ for diffeomorphisms $f, g$ and $u$ continuous, which is manifestly wrong in general (even for $g=$ id it only holds on the level of association; cf. [16], Prop. 1.2.70 (iv) and Sec. 3.2.2).

As there is no notion of manifold-valued distributions (see, however, section 4), and in the absence of additional structure, no growth conditions can be imposed on mappings between $X$ and $Y$; the maximal extension of $\mathcal{C}(X, Y)$ relevant to our present considerations is the space of locally bounded measurable mappings from $X$ to $Y$. In order to analyze it, we shortly recall some notions from measure theory on smooth manifolds (cf. [10]). Since zero sets are the same for any Lebesgue measure on $X$ there is a well-defined notion of Lebesgue-measurable subset of $X: A \subseteq X$ is Lebesgue measurable iff it can be written in the form $A=\bigcup_{j \in \mathbb{N}} K_{j} \cup N$ with $K_{j} \Subset X$ for all $j$ and $N$ a zero set. A mapping $u: X \rightarrow Y$ is called measurable if inverse images of Borel-measurable subsets of $Y$ under $u$ are Lebesgue measurable in $X$. For $X=\mathbb{R}^{m}, Y=\mathbb{R}^{n}$ this precisely reproduces the usual notion of Lebesgue measurability. Moreover, $u$ is Lebesgue measurable if and only if $\psi \circ u \circ \varphi^{-1}$ is Lebesgue measurable for any charts $\psi$ of $Y$ and $\varphi$ of $X$.

We define $\mathcal{L}_{\text {loc }}^{\infty}(X, Y)$ to be the set of all Lebesgue-measurable mappings $u: X \rightarrow$ $Y$ which are locally bounded in the following sense: For each $K \Subset X$ there exist $K^{\prime} \Subset Y$ and a zero set $N \subseteq X$ such that $u(K \backslash N) \subseteq K^{\prime}$. Factoring this space by the equivalence relation of coinciding Lebesgue-almost everywhere we obtain the space $L_{\text {loc }}^{\infty}(X, Y)$. In the particular case of $Y$ being a submanifold of some $\mathbb{R}^{s}$, $L_{\text {loc }}^{\infty}(X, Y)$ can be identified with the subset $\tilde{L}_{\text {loc }}^{\infty}(X, Y)$ of $L_{\text {loc }}^{\infty}\left(X, \mathbb{R}^{s}\right)$ (in the usual sense) whose elements possess a representative mapping $X$ into $Y$. Although in the $\mathbb{R}^{n}$-setting, $L_{\text {loc }}^{\infty}$ can be embedded (as a subspace of $\mathcal{D}^{\prime}$ ) into $\mathcal{G}$, the following example demonstrates that the construction given in Theorem 3.1 does not carry over to this setting in general:

3.3. Example. Let $X=\mathbb{R}, Y=S^{1} \subseteq \mathbb{R}^{2} \cong \mathbb{C}$ and let $u \in L_{\text {loc }}^{\infty}(X, Y)$ be given by $u(x)=(0,-1)$ for $x<0$ and $u(x)=(0,1)$ for $x>0$, i.e., $u(x)=i \operatorname{sgn}(x)$. Then with $\rho$ a standard mollifier as above, $u * \rho_{\varepsilon}(x)$ for $x \in \mathbb{R}$ covers the entire line connecting $-i$ and $i$. Therefore, it can never be contained in any tubular neighborhood of $S^{1}$ and the construction breaks down. 
Although the above example shows that one cannot embed $u$ using the construction involving tubular neighborhoods of $S^{1}$ this does not mean that one cannot construct an embedding of $L_{\text {loc }}^{\infty}\left(X, S^{1}\right)$ into $\mathcal{G}\left[X, S^{1}\right]$. In Example 3.3 it is fairly clear that one can obtain such an embedding by thinking of $S^{1}$ as a manifold with covering space $\mathbb{R}$. We will think of points in $S^{1}$ as lying in the interval $J=[0,2 \pi)$. Thus an element $u \in L_{\text {loc }}^{\infty}\left(X, S^{1}\right)$ defines a function $\hat{u}: X \rightarrow J \subset \mathbb{R}$ and hence an element of $L_{\mathrm{loc}}^{\infty}(X, \mathbb{R})$. This may be smoothed using a standard mollifier to give a family of smooth functions $\bar{u}_{\varepsilon}=\hat{u} * \rho$. Projecting this back to $S^{1}$ by defining $\tilde{u}_{\varepsilon}=\exp \left(i \bar{u}_{\varepsilon}\right)$ we define a family of smooth functions whose equivalence class defines an element $\tilde{u} \in \mathcal{G}\left[X, S^{1}\right]$. Furthermore it is clear that when applied to a smooth function this embedding gives the same result as $\sigma$. This construction may be generalized in an obvious way to give an embedding of $L_{\text {loc }}^{\infty}\left(X, T^{n}\right)$ into $\mathcal{G}\left[X, T^{n}\right]$.

A similar strategy may be applied to embed a function $u \in L_{\mathrm{loc}}^{\infty}(X, Y)$ where $Y$ is some compact Riemann surface apart from $S^{2}$. We have excluded the 2-sphere and have already dealt with the case of the torus, so the remaining Riemann surfaces have the structure $\mathbb{H}^{2} / \Gamma$, where $\mathbb{H}^{2}$ is the upper half-plane with the hyperbolic metric and $\Gamma$ is a properly discontinuous subgroup of $\operatorname{PSL}(2, \mathbb{R})$ that acts freely on $\mathbb{H}^{2}$. In this situation there exists some fundamental polygon $F$ with finitely many sides and for each side there is precisely one other side obtained by the action of some element $g \in \Gamma$ with different pairs of sides carried to each other by different elements of $\Gamma$ (see Th. 2.4.1 of 23 for details). By adding on precisely one side from each pair to the interior of $F$ we may obtain a region $D$ such that no two distinct elements of $D$ are related by the action of any element $g \in \Gamma$ and the sets $g D$ also cover $\mathbb{H}^{2}$. Given $u \in L_{\mathrm{loc}}^{\infty}(X, Y)$ we may now define a corresponding function $\hat{u}: X \rightarrow D \subset \mathbb{R}^{2}$ by defining $\hat{u}(x)$ to be the unique point in $D$ such that $u(x)=g \hat{u}(x)$. The function $\hat{u}$ is an element of $L_{\mathrm{loc}}^{\infty}\left(X, \mathbb{R}^{2}\right)$ and hence may be smoothed by convolution with a standard mollifier to give a family of smooth functions $\bar{u}_{\varepsilon}=\hat{u} * \rho_{\varepsilon}$. Projecting back down to $Y$ gives a family of smooth functions $\tilde{u}_{\varepsilon}$ whose equivalence class defines an element of $\mathcal{G}[X, Y]$. Again for the case of a smooth function the result of this embedding is the same as applying $\sigma$.

Turning now to the vector bundle setting we note that also in this situation we have a canonical embedding $\hat{\sigma}: \operatorname{Hom}(E, F) \rightarrow \operatorname{Hom}_{\mathcal{G}}(E, F), \hat{\sigma}: u \mapsto\left[(u)_{\varepsilon}\right]$. By $\operatorname{Hom}_{\mathcal{C}}(E, F)$ we denote the space of continuous vector bundle homomorphisms from $E$ to $F$. Using this notation we have:

3.4. Theorem. There exists an embedding $\hat{\iota}: \operatorname{Hom}_{\mathcal{C}}(E, F) \rightarrow \operatorname{Hom}_{\mathcal{G}}(E, F)$ with the following properties:

(i) $\hat{\imath}$ is a sheaf morphism.

(ii) $\left.\hat{\imath}\right|_{\operatorname{Hom}(E, F)}=\hat{\sigma}$.

(iii) Any representative of $\hat{\imath}(u)$ converges to $u$ uniformly on compact sets.

Proof. The idea is to first embed the base component by Theorem 3.1 and then employ a partition of unity argument in the fiber components adapting the construction of an embedding of $\mathcal{D}^{\prime}(X)$ into $\mathcal{G}(X)$ given in [27, Th. 2. To this end we shall make use of the technical apparatus developed in Theorem 2.5. Let $u \in \operatorname{Hom}_{\mathcal{C}}(E, F)$. With $\iota$ as in Theorem 3.1 we set $w \equiv\left[\left(w_{\varepsilon}\right)_{\varepsilon}\right]:=\iota(\underline{u})$. Choose now countable vector bundle atlases $\left\{\left(\Phi_{i}, U_{i}\right) \mid i \in \mathbb{N}\right\}$ of $E,\left\{\left(\Psi_{j}, V_{j}\right) \mid j \in \mathbb{N}\right\}$ of $Y$ such that for each $i$ there exists some $j$ with $\underline{u}\left(U_{i}\right) \subseteq V_{i_{j}}$. We may further suppose that each $U_{i}$ is relatively compact and choose a partition of unity $\left\{\chi_{i} \mid i \in \mathbb{N}\right\}$ on $X$ with $\operatorname{supp} \chi_{i} \subset U_{i}$ for each $i$. Let $\zeta_{i} \in \mathcal{D}\left(U_{i}\right)$ such that $\zeta_{i} \equiv 1$ on supp $\chi_{i}$ and choose 
some mollifier $\rho \in \mathcal{S}\left(\mathbb{R}^{m^{\prime}}\right)$ with unit integral and all higher moments vanishing. Since $w_{\varepsilon}$ converges to $\underline{u}$ uniformly on compact sets by Theorem 3.1, for each $i$ there exists some $\varepsilon_{i}$ such that

$$
w_{\varepsilon}\left(\operatorname{supp} \zeta_{i}\right) \subseteq V_{j_{i}}
$$

for $\varepsilon<\varepsilon_{i}$. Employing a gluing function as in the proof of Theorem 3.1 if necessary, we may suppose without loss of generality that (2) in fact holds for all $\varepsilon \in(0,1]$ and all $i \in \mathbb{N}$. Then define $v_{\varepsilon}^{i} \in \operatorname{Hom}_{\mathcal{C}}\left(\varphi_{i}\left(\left(\operatorname{supp} \zeta_{i}\right)^{\circ}\right) \times \mathbb{R}^{m^{\prime}}, \psi_{j_{i}}\left(V_{j_{i}}\right) \times \mathbb{R}^{n^{\prime}}\right)$ by

$$
(x, \xi) \mapsto\left(\psi_{j_{i}}\left(w_{\varepsilon}\left(\varphi_{i}^{-1}(x)\right)\right),\left(\left(\chi_{i} \circ \varphi_{i}^{-1}\right) \cdot\left(\Psi_{j_{i}} \circ u \circ \Phi_{i}^{-1}\right)^{(2)}\right) * \rho_{\varepsilon}(x) \cdot \xi\right),
$$

where convolution with $\rho_{\varepsilon}$ is to be read component-wise. Finally, we construct $v_{\varepsilon} \in \operatorname{Hom}(E, F)$ by

$$
v_{\varepsilon}:=\sum_{i \in \mathbb{N}} \zeta_{i} \bullet w_{\varepsilon} \Psi_{j_{i}}^{-1} \circ v_{\varepsilon}^{i} \circ \Phi_{i}
$$

and set $\hat{\imath}(u):=\left[\left(v_{\varepsilon}\right)_{\varepsilon}\right]$. Properties (i)-(iii) then follow from Theorem 3.1 and the proof of [27, Th. 2 .

3.5. Remark. It is in fact possible to embed vector bundle homomorphisms into $\operatorname{Hom}_{\mathcal{G}}(E, F)$, which are more singular (in the fiber component) than those considered in Theorem 3.4. To introduce such mappings we first recall an alternative description of smooth vector bundle homomorphisms $f: E \rightarrow F$. For vector bundle charts $\Phi_{\alpha}, \Phi_{\alpha^{\prime}}$ of $E$ and $\Psi_{\beta}, \Psi_{\beta^{\prime}}$ of $F$ we set $\Phi_{\alpha \alpha^{\prime}}:=\Phi_{\alpha} \circ \Phi_{\alpha^{\prime}}$ and analogously for $\Psi_{\beta \beta^{\prime}}$. It then follows from (1) that

$$
\begin{aligned}
& f_{\Psi_{\beta} \Phi_{\alpha}}^{(1)}(x)=\Psi_{\beta \beta^{\prime}}^{(1)}\left(f_{\Psi_{\beta^{\prime}} \Phi_{\alpha^{\prime}}}^{(1)}\left(\Phi_{\alpha^{\prime} \alpha}^{(1)}(x)\right)\right), \\
& f_{\Psi_{\beta} \Phi_{\alpha}}^{(2)}(x)=\Psi_{\beta \beta^{\prime}}^{(2)}\left(f_{\Psi_{\beta^{\prime}} \Phi_{\alpha^{\prime}}}^{(1)}\left(\Phi_{\alpha^{\prime} \alpha}^{(1)}(x)\right)\right) \cdot f_{\Psi_{\beta^{\prime}} \Phi_{\alpha^{\prime}}}^{(2)}\left(\Phi_{\alpha^{\prime} \alpha}^{(1)}(x)\right) \cdot \Phi_{\alpha^{\prime} \alpha}^{(2)}(x),
\end{aligned}
$$

so that we may identify smooth vector bundle homomorphisms $f: E \rightarrow F$ with families of smooth local vector bundle homomorphisms $f_{\Psi_{\beta} \Phi_{\alpha}}$ which transform according to (3), (4). As in the case of distributions on a manifold (cf. 19, Sec. 6.3) we may directly generalize the transformation behavior (3), (4) by allowing $f_{\Psi_{\beta} \Phi_{\alpha}}^{(2)}$ to be a matrix with distributional entries and by replacing compositions with distributional pullbacks. One restriction, however, immediately becomes apparent: $f_{\Psi_{\beta^{\prime}} \Phi_{\alpha^{\prime}}}^{(1)}$ has to be supposed smooth in order for the right hand side of (4) to be well defined (i.e., to avoid ill-defined products). This maximal class of distributional vector bundle homomorphisms (with smooth base component) can be embedded into $\operatorname{Hom}_{\mathcal{G}}(E, F)$ by a direct adaptation of the construction given in the proof of Theorem 3.4 .

\section{Manifold-VAlued Distributions}

Since distributions on manifolds are defined as continuous linear functionals (on the space of compactly supported densities), there is a priori no concept of distributions taking values in a differentiable manifold. In this section we propose the construction of a space $\mathcal{D}^{\prime}[X, Y]$ of distributions defined on $X$ and taking values in the manifold $Y$. The strategy is to define $\mathcal{D}^{\prime}[X, Y]$ as a quotient of a suitable subspace of $\mathcal{G}[X, Y]$.

We begin by analyzing the local situation. Sequential approaches to the theory of distributions in fact have a long history, dating back to [33]; see also [1] and 
38. The starting point for such considerations is the simple observation that the space of distributions is isomorphic to the quotient of the space of nets $\left(u_{\varepsilon}\right)_{\varepsilon}$ of smooth functions which converge in $\mathcal{D}^{\prime}$ modulo the space of nets with $u_{\varepsilon} \rightarrow 0$ distributionally. The Colombeau approach is of course sequential in nature and in fact it was noted already in [5] that $\mathcal{D}^{\prime}$ can equivalently be defined as a certain subspace of the Colombeau algebra $\mathcal{G}$. The most direct way of realizing $\mathcal{D}^{\prime}$ within the Colombeau framework is the following:

4.1. Lemma. Let $\Omega \subseteq \mathbb{R}^{n}$ be open and set

$$
\mathcal{A}(\Omega):=\left\{u=\left[\left(u_{\varepsilon}\right)_{\varepsilon}\right] \in \mathcal{G}(\Omega) \mid u_{\varepsilon} \text { converges in } \mathcal{D}^{\prime}(\Omega)\right\} .
$$

Call two elements $u, v$ equivalent, $u \equiv v$, if $u_{\varepsilon}-v_{\varepsilon} \rightarrow 0$ distributionally. Then $\mathcal{D}^{\prime}(\Omega)$ is linearly isomorphic to $\mathcal{A}(\Omega) / \equiv$.

Proof. With $\iota: \mathcal{D}^{\prime}(\Omega) \rightarrow \mathcal{G}(\Omega)$ the standard embedding, the map

$$
\begin{aligned}
\mathcal{D}^{\prime}(\Omega) & \hookrightarrow \mathcal{A}(\Omega) / \equiv \\
w & \rightarrow[\iota(w)]_{\equiv}
\end{aligned}
$$

is a linear isomorphism. In fact, $\iota(w)_{\varepsilon} \rightarrow w$ distributionally as $\varepsilon \rightarrow 0$. Linearity and injectivity are clear.

This result of course immediately generalizes to the case where the domain is a differentiable manifold (by employing the embedding provided by [27, Th. 1). However, when generalizing the target space to a smooth manifold $Y$, additional aspects have to be taken into account: most importantly, diffeomorphism invariance has to be implemented. Moreover, in the absence of additional structure it is not to be expected that unbounded distributions can be modelled (e.g., regularizations of the delta distribution have to display growth properties which can only be realized in the presence of scales). As similar obstacles have already been overcome in the construction of $\mathcal{G}[X, Y]$, in view of Lemma 4.1 the following definition provides a natural generalization:

\subsection{Definition. Let}

$$
\mathcal{A}[X, Y]:=\left\{u=\left[\left(u_{\varepsilon}\right)_{\varepsilon}\right] \in \mathcal{G}[X, Y] \mid \forall f \in \mathcal{C}^{\infty}(Y), f \circ u_{\varepsilon} \text { converges in } \mathcal{D}^{\prime}(X)\right\}
$$

and call $u, v \in \mathcal{A}[X, Y]$ model-associated, $u \approx_{\mathcal{M}} v$, if $f \circ u_{\varepsilon}-f \circ v_{\varepsilon} \rightarrow 0$ in $\mathcal{D}^{\prime}(X)$ for all $f \in \mathcal{C}^{\infty}(Y)$. The quotient space $\mathcal{D}^{\prime}[X, Y]:=\mathcal{A}[X, Y] / \approx_{\mathcal{M}}$ is called the space of distributions on $X$ taking values in $Y$.

The concept of model-association has been introduced in [26, Sec. 5 and has been compared with various other concepts of association. In particular, it was shown that $\approx_{\mathcal{M}}$ is strictly stronger than the usual concept of association in case $Y$ is a Euclidean space. Diffeomorphism invariance is implemented in the above definition through composition with smooth functions $f$ : any such $f$ can be viewed as an extension of a component of a chart; hence each $f \circ u_{\varepsilon}$ represents a different local picture of $u_{\varepsilon}$.

Our first observation concerning the above definition is that $u=\left[\left(u_{\varepsilon}\right)_{\varepsilon}\right] \in$ $\mathcal{A}[X, Y]$ implies that for each $f \in \mathcal{C}^{\infty}(Y)$ there exists some $u_{f} \in \mathcal{D}^{\prime}(X)$ such that $f \circ u_{\varepsilon} \rightarrow u_{f}$ in $\mathcal{D}^{\prime}(X)$. In fact, it turns out that $u_{f}$ has to be an element of $L_{\text {loc }}^{\infty}(X)$ : let $K$ be a compact subset of $X$. Then by the c-boundedness of $\left(u_{\varepsilon}\right)_{\varepsilon}$ it follows that $\left(f \circ u_{\varepsilon}\right)_{\varepsilon}$ is uniformly bounded on $K$, hence possesses a weak-* convergent subsequence $\left(\left.f \circ u_{\varepsilon_{k}}\right|_{K}\right)_{k}$ with limit $v_{f} \in L^{\infty}(K)$. Therefore $\left.u_{f}\right|_{\mathcal{D}(K)}=v_{f}$ 
and the claim follows by covering $X$ with relatively compact open sets. Moreover, from Theorem 3.1 (iii) it follows that $\mathcal{C}(X, Y)$ can be embedded as a subspace into $\mathcal{D}^{\prime}[X, Y]$ and that for $u \in \mathcal{C}(X, Y)$, each $u_{f}$ is a continuous function.

For the special case $Y=\mathbb{R}$ and $f=\operatorname{id}_{\mathbb{R}}$ it follows that if a sequence $\left(u_{\varepsilon}\right)_{\varepsilon} \in$ $\mathcal{A}[X, \mathbb{R}]$, then $\left(u_{\varepsilon}\right)_{\varepsilon}$ converges to an element $u \in L_{\text {loc }}^{\infty}(X)$ distributionally. Moreover, if we denote by $\iota_{S}$ a sheaf embedding of $\mathcal{D}^{\prime}(X)$ into $\mathcal{G}(X)$ as in [16], Thm. 3.2 .10 , we see that if $w \in \mathcal{D}^{\prime}(X), \iota_{S}(w)=\left[\left(w_{\varepsilon}\right)_{\varepsilon}\right]$ with $\left(w_{\varepsilon}\right)_{\varepsilon} \in \mathcal{A}[X, \mathbb{R}]$, then $w \in L_{\text {loc }}^{\infty}(X)$. Hence representatives of elements of $\mathcal{D}^{\prime}[X, \mathbb{R}]$ which come from classical distributions actually are $L_{\mathrm{loc}}^{\infty}(X)$-functions.

From these considerations one might be led to believe that $\mathcal{D}^{\prime}[X, Y]$ singles out a certain subspace of $L_{\mathrm{loc}}^{\infty}(X, Y)$ in the sense that for each $u \in \mathcal{A}[X, Y]$ there should exist some underlying $v \in L_{\mathrm{loc}}^{\infty}(X, Y)$ with $u_{f}=f \circ v$ for all $f \in \mathcal{C}^{\infty}(Y)$. However, as the following example demonstrates, the situation is more involved:

4.3. Example. Let $X=Y=\mathbb{R}$ and consider the net $u_{\varepsilon}(x)=\sin (x / \varepsilon)$. As is easily verified, each $u_{\varepsilon}^{j}\left(j \in \mathbb{N}_{0}\right)$ converges in $\mathcal{D}^{\prime}(\mathbb{R})$. Hence every polynomial in $u_{\varepsilon}$ converges in $\mathcal{D}^{\prime}$ and by the Weierstrass approximation theorem it follows that $\left(u_{\varepsilon}\right)_{\varepsilon} \in \mathcal{A}[X, Y]$ (in fact, $f \circ u_{\varepsilon}$ converges in $\mathcal{D}^{\prime}$ for all continuous $f$ ). Nevertheless, there does not exist any $v \in L_{\text {loc }}^{\infty}(\mathbb{R})$ such that $f \circ u_{\varepsilon} \rightarrow f \circ v$ for all $f \in \mathcal{C}^{\infty}(\mathbb{R})$. Otherwise, the choice of $f=$ id would entail $v=0$, whereas for $f(x)=x^{2}, f \circ u_{\varepsilon} \rightarrow$ $1 / 2$, a contradiction.

In case $Y=\mathbb{R}^{n}$, a local description of elements of $\mathcal{D}^{\prime}[X, Y]$ can be given in terms of Young measures (cf., e.g., [14, p. 16, Th. 11). In fact, in this situation we may w.l.o.g. (using charts) suppose that $X=U$ is a bounded open subset of $\mathbb{R}^{m}$ and that for $\left[\left(u_{\varepsilon}\right)_{\varepsilon}\right] \in \mathcal{D}^{\prime}\left[U, \mathbb{R}^{n}\right],\left\{u_{\varepsilon} \mid \varepsilon \in I\right\}$ is uniformly bounded (by choosing $\left.f=\operatorname{pr}_{j}\right)$. Hence there exists a subsequence $\left(u_{\varepsilon_{k}}\right)_{k}$ and a Young measure $\left(\nu_{x}\right)_{x \in U}$ such that for each $f \in \mathcal{C}(Y)$,

$$
f \circ u_{\varepsilon_{k}}(x) \rightarrow \int_{\mathbb{R}^{m}} f(y) d \nu_{x}(y)
$$

weak-* in $L^{\infty}(U)$.

Thus elements of $\mathcal{D}^{\prime}\left[X, \mathbb{R}^{n}\right]$ do not possess an underlying description by an $L_{\text {loc }}^{\infty}$ function (as shown by Example 4.3) but they do possess a description in terms of Young measures in the above sense.

To conclude this section, we turn to the question of stability of $\mathcal{D}^{\prime}[X, Y]$ under differentiation. We first note that differentiating an element of $\mathcal{G}[X, Y]$ gives a generalized vector bundle homomorphism of the respective tangent bundles rather than simply an element of $\mathcal{G}[X, Y]$ (see [24]). Hence when considering stability under differentiation we need to take into account this change of category.

As a preparatory result, we provide an alternative description of vb-equivalence for generalized vector bundle homomorphisms of tangent bundles:

4.4. Lemma. Let $E=T X, F=T Y$ and $u, v \in \mathcal{E}_{M}^{\mathrm{VB}}[T X, T Y]$. Then the following statements are equivalent:

(i) $u \sim_{v b} v$.

(ii) $T f \circ u \sim_{v b} T f \circ v$ in $\mathcal{E}_{M}^{\mathrm{VB}}\left[T X, \mathbb{R} \times \mathbb{R}^{n}\right]$ for all $f \in \mathcal{C}^{\infty}(Y)$.

Proof. We first note that by [29], Th. 4.2 it suffices to prove the equivalence for $\sim_{v b 0}$ instead of $\sim_{v b}$ (i.e., with $k=0$ in Definition 1.3 (b) (ii)). Moreover, the same result establishes (i) $\Rightarrow$ (ii) (even for general vector bundle homomorphisms 
instead of tangent maps). Conversely, property (i) of Definition 1.3 (b) follows from Theorem 1.2 (ii) since for $\varphi$ a chart of $X$ (hence $T \varphi$ a vector bundle chart of $T X)$ we have

$$
\left(T f \circ u_{\varepsilon}-T f \circ v_{\varepsilon}\right)_{\mathrm{id} T \varphi}^{(1)}(\varphi(p))=\left(f \circ \underline{u_{\varepsilon}}-f \circ \underline{v_{\varepsilon}}\right) \circ \varphi^{-1}(p) .
$$

For establishing property (ii) it suffices to note that any vector bundle chart $T \psi$ of $T Y$ is the restriction of some $T f$ for a suitable extension of $\psi$ (cf. the proof of Prop. 4.1 in 29]).

The above result suggests that to test equivalence of tangent maps of elements of $\mathcal{D}^{\prime}[X, Y]$ one should compose with tangent maps of elements of $\mathcal{C}^{\infty}(Y)$. This leads to the following.

4.5. Definition. Two elements $u, v$ of $\operatorname{Hom}_{\mathcal{G}}(T X, T Y)$ are called model-vbequivalent if $T f \circ u_{\varepsilon}-T f \circ v_{\varepsilon}$ converges to 0 distributionally for all $f \in \mathcal{C}^{\infty}(Y)$.

More precisely, this means that for any chart $(\varphi, V)$ of $X$ and any $f \in \mathcal{C}^{\infty}(Y)$ we have

$$
\begin{aligned}
& f \circ u_{\varepsilon} \circ \varphi^{-1}-f \circ v_{\varepsilon} \circ \varphi^{-1} \rightarrow 0, \\
& D\left(f \circ u_{\varepsilon} \circ \varphi^{-1}-f \circ v_{\varepsilon} \circ \varphi^{-1}\right) \rightarrow 0
\end{aligned}
$$

in $\mathcal{D}^{\prime}(\varphi(V))$. Since distributional convergence is stable under derivatives it therefore follows that for $u, v \in \mathcal{A}[X, Y]$, model equivalence entails vb-model equivalence of the respective tangent maps. As in the local distributional setting, stability under differentiation can therefore be achieved also for $\mathcal{D}^{\prime}[X, Y]$ if iterated tangent maps of elements of $\mathcal{C}^{\infty}(Y)$ are used for testing equivalence of derivatives.

\section{ACKNOWLEDGMENTS}

We would like to thank James D.E. Grant for several helpful discussions.

\section{REFERENCES}

[1] Antosik, P., Mikusiński, J., Sikorski, R. Theory of Distributions. The Sequential Approach. Elsevier, 1973. MR0365130(51:1383)

[2] Aragona, J., Biagioni, H. A. Intrinsic definition of the Colombeau algebra of generalized functions. Analysis Mathematica, 17:75-132, 1991. MR.1198956 (94d:46043)

[3] Biagioni, H. A. A Nonlinear Theory of Generalized Functions, volume 1421 of Lecture Notes in Mathematics. Springer, Berlin, 1990. MR1049623 (91g:46047)

[4] Colombeau, J. F. New Generalized Functions and Multiplication of Distributions. NorthHolland, Amsterdam, 1984. MR738781 (86c:46042)

[5] Colombeau, J. F. Elementary Introduction to New Generalized Functions. North-Holland, Amsterdam, 1985. MR808961 (87f:46064)

[6] Colombeau, J. F. Multiplication of distributions. Bull. Amer. Math. Soc. (N.S.), 23:251-268, 1990. MR:1028141 (91c:46053)

[7] Colombeau, J. F., Meril, A. Generalized functions and multiplication of distributions on $\mathcal{C}^{\infty}$ manifolds. J. Math. Anal. Appl., 186:357-364, 1994. MR.1292997 (95f:46060)

[8] De Rham, G. Differentiable Manifolds, volume 266 of Grundlehren der mathematischen Wissenschaften. Springer, Berlin, 1984. MR.760450 (85m:58005)

[9] De Roever, J. W., Damsma, M. Colombeau algebras on a $\mathcal{C}^{\infty}$-manifold. Indag. Mathem., N.S., 2(3), 1991. MR 1149687 (93e:46046)

[10] Dieudonné, J. Treatise on Analysis, volume 3. Academic Press, New York, 1972. MR0350769 (50:3261) 
[11] Dapić, N.; Pilipović, S. Microlocal analysis of Colombeau's generalized functions on a manifold. Indag. Math. (N.S.) 7 no. 3, 293-309, 1996. MR.1621385 (99h:46065)

[12] Dapić, N.; Pilipović, S.; Scarpalezos, D. Microlocal analysis of Colombeau's generalized functions: Propagation of singularities. J. Anal. Math. 75 51-66, 1998. MR1655823 (99k:35007)

[13] Delcroix, A., Hasler, M., Pilipović, S., Valmorin, V. Generalized function algebras as sequence space algebras. Proc. Amer. Math. Soc. 132 (2004), no. 7, 2031-2038 MR2053975 (2004m:46009)

[14] Evans, L. C. Weak convergence methods for nonlinear partial differential equations, volume 74 of CBMS Regional Conference Series in Mathematics. 1990. MR1034481 (91a:35009)

[15] Grosser, M., Farkas, E., Kunzinger, M., Steinbauer, R. On the foundations of nonlinear generalized functions I, II. Mem. Amer. Math. Soc., 153(729), 2001. MR1848157(2002f:46066)

[16] Grosser, M., Kunzinger, M., Oberguggenberger, M., Steinbauer, R. Geometric Theory of Generalized Functions, volume 537 of Mathematics and its Applications 537. Kluwer Academic Publishers, Dordrecht, 2001.

[17] Grosser, M., Kunzinger, M., Steinbauer, R., Vickers, J. A global theory of algebras of generalized functions. Adv. Math., 166:179-206, 2002. MR1882858 (2003a:46108)

[18] Hirsch, M. W. Differential topology, volume 33 of Graduate Texts in Mathematics. SpringerVerlag, New York, 1994. MR1336822 (96c:57001)

[19] Hörmander, L. The Analysis of Linear Partial Differential Operators I, volume 256 of Grundlehren der mathematischen Wissenschaften. Springer, Berlin, 1990. MR1065993 (91m:35001a)

[20] Jelínek, J. An intrinsic definition of the Colombeau generalized functions. Comment. Math. Univ. Carolinae, 40:71-95, 1999. MR 1715203 (2000i:46034)

[21] Jelínek, J. On introduction of two diffeomorphism invariant Colombeau algebras. Comment. Math. Univ. Carolinae 45 (2004), no. 4, 615-632. MR2103080(2005k:46098a)

[22] Jelínek, J. Equality of two diffeomorphism invariant Colombeau algebras. Comment. Math. Univ. Carolinae 45 (2004), no. 4, 633-662. MR2103081(2005k:46098b)

[23] Jost, J. Compact Riemann surfaces. Springer, Berlin, 1997. MR1632873 (2000k:32011)

[24] Kunzinger, M. Generalized functions valued in a smooth manifold. Monatsh. Math., 137:3149, 2002. MR 1930994 (2003h:46109)

[25] Kunzinger, M., Oberguggenberger, M. Group analysis of differential equations and generalized functions. SIAM J. Math. Anal., 31(6):1192-1213, 2000. MR.1766563 (2001c:35010)

[26] Kunzinger, M., Oberguggenberger, M., Steinbauer, R., Vickers, J. Generalized flows and singular ODEs on differentiable manifolds. Acta Appl. Math., 80:221-241, 2004. MR 2035508 (2004k:46064)

[27] Kunzinger, M., Steinbauer, R. Foundations of a nonlinear distributional geometry. Acta Appl. Math., 71:179-206, 2002. MR1914741 (2003e:46125)

[28] Kunzinger, M., Steinbauer, R. Generalized pseudo-Riemannian geometry. Trans. Amer. Math. Soc., 354(10):4179-4199, 2002. MR1926870 (2003g:46046)

[29] Kunzinger, M., Steinbauer, R., Vickers, J. Intrinsic characterization of manifold-valued generalized functions. Proc. London Math. Soc., 87(2):451-470, 2003. MR.1990935 (2004e:46092)

[30] Lee, J. M. Introduction to smooth manifolds, volume 218 of Graduate Texts in Mathematics. Springer-Verlag, New York, 2003. MR.1930091 (2003k:58001)

[31] Marsden, J. E. Generalized Hamiltonian mechanics. Arch. Rat. Mech. Anal., 28(4):323-361, 1968. MR0224935(37:534)

[32] Marti, Jean-Andre $(\mathcal{C}, \mathcal{E}, \mathcal{P})$-sheaf structures and applications. Nonlinear theory of generalized functions (Vienna, 1997), 175-186, Chapman \& Hall, Boca Raton, FL, 1999. MR 1699819 (2000f:46050)

[33] Mikusiński, J. Sur la méthode de généralisation de M. Laurent Schwartz et sur la convergence faible. Fund. Math., 35:235-239, 1948. MR0027958(10:382d)

[34] Nash, J. The imbedding problem for Riemannian manifolds. Ann. of Math. (2) 63 (1956), 20-63. MR0075639(17:782b)

[35] Oberguggenberger, M. Multiplication of Distributions and Applications to Partial Differential Equations, volume $\mathbf{2 5 9}$ of Pitman Research Notes in Mathematics. Longman, Harlow, U.K., 1992. MR1187755 (94d:46044)

[36] Schwartz, L. Sur l'impossibilité de la multiplication des distributions. C. R. Acad. Sci. Paris, 239:847-848, 1954. MR0064324 (16:265e) 
[37] Steinbauer, R., Vickers, J. The use of generalized functions and distributions in general relativity. Classical Quantum Gravity 23 (2006), no. 10, R91-R114. MR2226026 (2007d:83018)

[38] Temple, G. Theories and applications of generalized functions. J. London Math. Soc., 28:134148, 1953. MR0054847(14:989a)

Department of Mathematics, University of Vienna, Nordbergstr. 15, A-1090 Wien, AUSTRIA

E-mail address: michael.kunzinger@univie.ac.at

Department of Mathematics, University of Vienna, Nordbergstr. 15, A-1090 Wien, AUSTRIA

E-mail address: roland.steinbauer@univie.ac.at

Faculty of Mathematical Studies, University of Southampton, Highfield, SouthampTON, SO17 1BJ, United Kingdom

E-mail address: J.A.Vickers@maths.soton.ac.uk 